\title{
Prevention of Decline in Cognition after Stroke Trial (PODCAST): a study protocol for a factorial randomised controlled trial of intensive versus guideline lowering of blood pressure and lipids
}

Daniel J Blackburn ${ }^{1}$, Kailash Krishnan², Lydia Fox², Clive Ballard ${ }^{3}$, Alistair Burns ${ }^{4}$, Gary A Ford ${ }^{5}$, Jonathan Mant ${ }^{6}$, Peter Passmore ${ }^{7}$, Stuart Pocock ${ }^{8}$, John Reckless ${ }^{9}$, Nikola Sprigg ${ }^{2}$, Rob Stewart ${ }^{10}$, Joanna Wardlaw ${ }^{11}$ and Philip MW Bath²

\begin{abstract}
Background: Stroke is a common cause of cognitive impairment and dementia. However, effective strategies for reducing the risk of post-stroke dementia remain undefined. Potential strategies include intensive lowering of blood pressure and/or lipids.

Methods/Design: Design: multi-centre prospective randomised open-label blinded-endpoint controlled partial-factorial phase IV trial in secondary and primary care.

Participants: 100 participants from 30 UK Stroke Research Network sites who are post- ischemic stroke or intracerebral haemorrhage by three to seven months.

Interventions - all patients (1:1): intensive versus guideline blood pressure lowering (target systolic $<125 \mathrm{mmHg}$ versus $<140 \mathrm{mmHg}$ ).

Interventions - ischemic stroke (1:1): intensive versus guideline lipid lowering (target low density lipoprotein-cholesterol (LDL-c) $<1.4 \mathrm{mmol} / \mathrm{I}$ versus $<3 \mathrm{mmol} / \mathrm{I})$.

Hypotheses: does 'intensive' blood pressure lowering therapy and/or 'intensive' lipid control reduce cognitive decline and dementia in people with ischemic stroke; and does 'intensive' blood pressure lowering therapy reduce cognitive decline and dementia in patients with hemorrhagic stroke.

Primary outcome: Addenbrooke's Cognitive Examination-Revised.

Secondary outcomes: feasibility of recruitment and retention of participants, tolerability and safety of the interventions, achieving and maintaining the blood pressure and lipid targets, maintaining differences in systolic blood pressure (> $10 \mathrm{mmHg}$ ) and low density lipoprotein-cholesterol (> $1 \mathrm{mmol} / \mathrm{l})$ between the treatment groups, and performing clinic and telephone follow-up of cognition measures.

Randomisation: using stratification, minimization and simple randomization.

Blinding: participants receive open-label management. Cognition is assessed both unblinded (in clinic) and blinded (by telephone) to treatment. Adjudication of events (dementia, vascular, serious adverse events) is blinded to management.

Discussion: The PODCAST trial is ongoing with 78 patients recruited to date from 22 sites. Outcomes of cognitive impairment and dementia are accruing.
\end{abstract}

Trial registration: ISRCTN85562386

Keywords: Stroke, Post-stroke cognitive impairment, Post-stroke dementia, Blood pressure lowering, Lipid lowering

\footnotetext{
* Correspondence: philip.bath@nottingham.ac.uk

${ }^{2}$ Stroke Trials Unit, Division of Clinical Neuroscience, University of Nottingham, City Hospital campus, Nottingham NG5 1PB, UK

Full list of author information is available at the end of the article
} 


\section{Background}

\section{Post-stroke cognitive impairment}

Post-stroke cognitive impairment is common, ranging from 17 to 92\%, [1,2] and is associated with increased mortality and decreased quality of life [3-5]. Nevertheless, cognitive impairment may improve or deteriorate following a stroke [6]. Risk factors for cognitive decline include executive dysfunction, white matter hyperintensities (WMH), ApoE e4 status [7] and atrophy of crucial brain areas [8].

Many potential interventions for preventing cognitive decline have been proposed, including blood pressure (BP) and lipid lowering, antiplatelet agents, anti-oxidant vitamins, and cholinesterase inhibitors. Of these, lowering $\mathrm{BP}$ and blood lipids are priorities for testing.

\section{Blood pressure lowering}

Lowering BP post-stroke is highly effective in reducing recurrent and other vascular events, as shown in individual trials (such as Post-stroke Antihypertensive Treatment Study (PATS, $\mathrm{n}=5,665$ ) and Perindopril protection against recurrent stroke study (PROGRESS, $\mathrm{n}=6,105$ ) $[9,10]$ ) and a meta-analysis of them [11]. However, the effect on cognitive function of lowering BP is far less clear.

Longitudinal studies have shown that premorbid high systolic blood pressure (SBP) and diastolic blood pressure (DBP) are associated with WMH and an increased risk of Alzheimer's disease (AD) and vascular dementia [12-14]. Although no trials have been expressly designed to test the effect of lowering BP on subsequent cognition poststroke, several have included cognition as a secondary outcome measure. Whilst potential benefit was seen in the PROGRESS study [15], none was found in the Prevention Regimen for Effectively Avoiding Second Strokes trial (PRoFESS, $n=20,332$ ) [16]. Similar mixed results have been seen in trials of BP lowering in non-stroke populations, for example, the Hypertension in the Very Elderly Trial (HYVET, $\mathrm{n}=3,845$ ) and Systolic Hypertension in Europe (Syst-Eur, $n=4,695$ ) $[17,18]$. In a meta-analysis involving both stroke and non-stroke patients, lowering BP was associated with less cognitive decline, and a trend to less dementia [19]; meta-regression suggested that the degree of reduction in cognition was related to the magnitude of BP lowering.

The Secondary Prevention of Small Subcortical Strokes (SPS3, $\mathrm{n}=3,020$ ) factorial trial of intensive versus guideline BP lowering, and aspirin/clopidogrel versus aspirin [20], in patients with MRI-proven lacunar stroke will be presenting the effects of intensive BP lowering on cognition [21] in 2014. The ongoing PRESsure in established cERebral small VEssel disease (PRESERVE, $\mathrm{n}=422$ ) trial is also investigating the effect of lowering $\mathrm{BP}$ in patients with established cerebral small vessel disease (http://www.
controlled-trials.com/ISRCTN37694103, downloaded 20 June 2013).

\section{Lipid lowering}

The majority of information on lipid lowering and cognition relates to statins rather than older interventions such as fibrates, nicotinic acid derivatives or resins. Statins have pleiotropic effects that include lowering cholesterol (specifically low density lipoprotein-cholesterol (LDL-c)) and reducing platelet activity, inflammation and the release of cytokines and acute phase reactants [22,23]. These effects might limit the progression of Alzheimer's pathology from an asymptomatic state to symptomatic, or deterioration after stroke [24]. Although statins are one of the most widely prescribed drugs with clear health benefits in reducing vascular events, including stroke [25-27], and death, there is little direct evidence that lipid lowering prevents cognitive decline in either people with normal cognition or patients with cognitive impairment.

The Heart Protection Study (HPS, $n=20,536$ ) found significant reductions in coronary artery and cerebrovascular events with simvastatin [28] but there was no difference in cognition on treatment (baseline measures were not taken so change could not be assessed), assessed using the Telephone Interview for Cognitive Status (TICS), even when sub-groups of older patients, and those with prior stroke, were analysed. Similarly, the Pravastatin in elderly individuals at risk of vascular disease (PROSPER, $\mathrm{n}=5,804$ ) trial in people aged 70 to 82 with vascular risk factors reported no effect on cognition (measured using Mini Mental State Examination (MMSE), Stroop and a series of psychometric tests) [29]. A meta-analysis of three trials found a nonsignificant trend to higher MMSE scores in patients with $\mathrm{AD}$ who were randomised to statin treatment (atorvastatin, simvastatin) [19].

Thus, there is no clear evidence that statins reduce the risk of cognitive decline or dementia but this has not been formally examined in a high-risk population. In contrast, there has been some concern that statins are associated with reversible cognitive impairment [30], either due to an idiosyncratic response to statins or an underlying mitochondrial dysfunction.

\section{Methods/design \\ Purpose}

To develop interventions to prevent cognitive decline and dementia after stroke.

\section{Primary objectives \\ Start-up phase}

To determine the feasibility of recruiting and retaining patients, and identify any barriers to achieving BP and lipid targets. 


\section{Main phase}

To determine if 'intensive' blood pressure lowering therapy, and/or 'intensive' lipid lowering therapy, after stroke reduces cognitive decline and dementia.

\section{Secondary objectives Start-up phase}

To determine the feasibility of recruiting and retaining sites, reaching and maintaining target $\mathrm{BP}$ and lipid levels, performing cognitive assessment in clinic and by telephone, and the tolerability and safety of the management strategies.

\section{Main phase}

To determine if 'intensive' blood pressure lowering therapy, and/or 'intensive' lipid lowering therapy, after stroke reduces poor quality of life, poor function, depression, stroke recurrence, vascular events, and death.

\section{Aims}

\section{Start-up phase}

This is assessing the:

- Ability to deliver the protocol

- Ability to recruit 30 recruiting sites

- Ability to recruit and retain 600 participants

- Ability to achieve and maintain differences in systolic $\mathrm{BP} \geq 10 \mathrm{mmHg}$ and LDL-c $\geq 1 \mathrm{mmol} / \mathrm{l}$ between the 'intensive' and 'guideline' treatment groups

- Ability to perform clinic and telephone follow-up of outcome measures

- Sensitivity of the Addenbrooke's Cognitive Examination-R (ACE-R) and other cognitive measures to change over time

- Tolerability and safety of the intervention

\section{Main phase}

A main phase was planned to assess the safety and efficacy of intensive versus guideline $\mathrm{BP}$ and lipid management in preventing cognitive decline. A total of 3,400 patients (start-up 600, main 2,800) post-stroke were planned. However, the main phase was cancelled 24 months into the pilot phase because of a failure to achieve a sufficiently high recruitment rate.

\section{Design}

PODCAST is a multi-centre prospective randomised open-label blinded-endpoint controlled partial-factorial phase IV trial. The study is conducted according to the principles of the Declaration of Helsinki and 'International Conference on Harmonisation of Good Clinical Practice'. Study approval by national (UK, approval 09/H0403/71, date 12 November 2009) and local research ethics committees (all centres) has been obtained. As a management trial, the study does not fall under the remit of the UK Medicines and Healthcare Products Regulatory Authority (as confirmed by them). The management of personal data adheres to the UK Data Protection Act 1998. The UK National Institutes Health Research Stroke Research Network supports the trial through screening and recruitment of patients (23 September 2009).

\section{Patient population}

Participants are recruited from hospital-based stroke services, and are consented for a face-to face assessment of cognition (telephone-Mini Mental Status Examination, t-MMSE) and function (modified Rankin Scale, $\mathrm{mRS})$ at 8 to 26 weeks after the stroke. If the participant is eligible and interested after the initial assessment, fasting lipids, glucose, urea and electrolytes, and HbA1c are tested.

\section{Inclusion criteria}

1. Age $>70$ years and $\mathrm{t}-\mathrm{MMSE}>16$ (maximum score 22 ); or age $>60$ years and t-MMSE 17 to 20

2. Functionally independent (mRS 0 to 2).

3. Ischemic stroke (IS, any Oxfordshire Community Stroke Project or Trial of Org10172 in Acute Stroke Treatment type [31,32]) or spontaneous intracerebral haemorrhage.

4. Three to seven months post-event (to allow cognitive, neurological, BP and lipid stabilisation [33], but avoid attrition).

5. Systolic BP 125 to $170 \mathrm{mmHg}$.

6. Total cholesterol (TC) 3 to $8 \mathrm{mmol} / \mathrm{l}$.

7. Presence of an informant (ideally two): partner, sibling, child, friend (for Informant Questionnaire on Cognitive Decline in the Elderly, IQCODE [34]).

8. Capacity and willingness to give consent.

\section{Exclusion criteria}

1. Participants not meeting inclusion criteria.

2. Subarachnoid haemorrhage.

3. Secondary intracranial haemorrhage (trauma, arterio-venous malformation, cavernoma).

4. No CT/MR brain scan within ten days of index stroke.

5. Inability to give consent or do study measures, for example, severe dysphasia, weakness of dominant arm.

6. Profound deafness.

7. Severe hypertension (systolic BP $>170 \mathrm{mmHg}$ ).

8. Definite need for 'intensive' BP control.

9. Severe hypercholesterolemia (TC $>8 \mathrm{mmol} / \mathrm{l}$ ).

10. Definite need for, or demonstrated intolerance of, 'high intensity' statin. 
11. Definite need for a cholinesterase inhibitor for dementia.

12. Familial stroke associated with dementia, for example, CADASIL.

13. Chronic renal failure: eGFR $<45$ (or eGFR $<37$ in people of African/Afro-Caribbean origin).

14. Liver disease, ALT > three times upper limit of normal, using local laboratories ranges.

15. Ongoing participation in trials involving drug and/ or devices. Participants already in another trial may be recruited to PODCAST, provided that participation in the other trial is complete prior to PODCAST randomisation.

16. Any serious medical co-morbidity (for example, active malignancy) such that the life expectancy is $<24$ months.

17. Clinically unstable at the time of enrollment.

18. Dementia.

\section{Informed consent}

All participants must have capacity, and be willing and able to provide written informed consent. Participants are screened for potential recruitment during their initial presentation to the hospital stroke services, and are given an information sheet explaining the study.

\section{Screening consent}

Informed consent for formal screening is taken in hospital for conducting the following assessments, 8 to 26 weeks after their stroke:

- Assessment of cognition - t-MMSE

- Assessment of function - mRS

- Blood test - fasting lipids, glucose, urea and electrolytes, HbA1c

The availability of an informant (partner, sibling, child or friend), ideally with a backup, is key. Informants provide information on the participant's prior cognitive state and decline (via the IQCODE).

Both the patient and informant are then given information sheets to take away and review.

\section{Full consent}

Providing the patient fulfils the inclusion-exclusion criteria at the screening visit, full consent is taken at the baseline visit. This includes an assessment of capacity by telling the patient about the trial and then asking them to answer questions based on this information:

- What condition? Stroke

- What is the trial trying to prevent? Dementia

- What are the interventions? Intensive BP and/or lipid lowering
Following any questions about the trial, written informed consent of both the patient and informant is then performed. Patients may also give consent for two sub-studies:

- Ambulatory BP monitoring

- On-treatment CT scan

Part of the consent process involves both the patient and informant agreeing to the latter assuming the right of proxy consent if the patient loses capacity during the trial.

\section{Randomisation}

Eligible and consenting participants are randomised centrally using a secure internet site in real-time: https:// www.nottingham.ac.uk/ nszwww/podcast/podcasttrialdb/ podcast_login.php.

The process of randomisation includes stratification, minimisation and simple randomisation, based on information gathered by the local recruiting investigators. Stratification and minimisation allow for improved matching at baseline: stratification allows variable categories to be treated as nested trials in their own right; minimisation increases statistical power [35]. Simple randomisations reduce predictability. The minimisation variables will be used for adjustment of the primary and secondary analyses.

\section{Stratification}

- Stroke type (IS, spontaneous ICH)

Patients with IS are randomised to both BP lowering (intensive versus guideline) and lipid lowering (intensive versus guideline) strategies.

Patients with $\mathrm{ICH}$ are randomised to BP lowering (intensive versus guideline) strategy only.

\section{Minimisation (on key prognostic/logistical variables)}

- Age $(<70 />70$ years $)$

- Sex (female, male)

- Dysphasia (no, yes)

- Cognition, ACE-R $(\geq 85 /<85)$

- Systolic BP $(<150 />150 \mathrm{mmHg})$

- Total cholesterol $(<4.0 / \geq 4.0 \mathrm{mmol} / \mathrm{L})$

- Function/dependency, mRS $(0 / \geq 1)$

- Brain region (subcortex/cortex)

- Evidence of periventricular white matter lucency (no, yes)

- Time since index stroke (<140/> 140 days)

- Number of antihypertensive drugs $(<2 / \geq 2)$

- Already on a statin (yes, no) 


\section{Simple randomisation}

On $5 \%$ of patients at time of minimisation.

\section{Randomisation groups}

Study participants are randomized to:

- Intensive versus guideline BP lowering - all participants

- Intensive versus guideline lipid lowering - ischaemic stroke only

As a result, patients can be randomised to one of six groups:

1. Intensive BP lowering and intensive lipid lowering (ischaemic stroke only)

2. Intensive BP lowering and guideline lipid lowering (ischaemic stroke only)

3. Intensive BP lowering only (ICH only)

4. Guideline BP lowering and intensive lipid lowering (ischaemic stroke only)

5. Guideline BP lowering and guideline lipid lowering (ischaemic stroke only)

6. Guideline BP lowering only (ICH only)

Assuming that approximately $10 \%$ of patients will be enrolled with $\mathrm{ICH}$, the distribution of patients between the six treatment groups will, for every 100 patients, approximate to $22.5 \%$ in each of the four groups of ischaemic stroke patients (intensive versus guideline BP; intensive versus guideline lipid) and $5 \%$ in each group of patients with ICH (intensive versus guideline BP).

\section{Interventions}

The trial is assessing management strategies ('intensive' versus 'guideline') rather than particular drugs. All participants receive standard lifestyle advice and rehabilitation (as per NICECG 68, 2008 [36]) including: diet, exercise, smoking advice, rehabilitation, psychological assessment and therapy, modification of all risk factors and other relevant interventions.

\section{Guideline management}

Participants randomised to the guideline groups are managed by their general practitioner (GP) who follows national/international guidelines and local practice.

\section{Guideline BP lowering}

It is expected that GPs will aim for a systolic $\mathrm{BP}<$ $140 \mathrm{mmHg}$.

\section{Guideline lipid lowering}

It is expected that GPs will aim for a LDL-c $<3 \mathrm{mmol} / \mathrm{l}$ (or $\mathrm{TC}<5 \mathrm{mmol} / \mathrm{l}$ ).

\section{Intensive management}

Participants in the intensive group are managed by the local hospital stroke research team and medications initiated by the local investigator and continued by the GP. The trial does not stipulate specific drugs but gives examples of drugs (and relevant doses) to use from the different drug classes. Guidance on which drugs to start and add, how to titrate, and how to manage participants with various contra-indications to medications, are included in algorithms; these are updated to include new information as relevant.

Intensive $\mathrm{BP}$ and lipid management strategies may be attenuated or stopped if the patient or their informant withdraws consent, for safety, or if unacceptable adverse events develop. If the participant wishes to withdraw from treatment, they are requested to permit primary outcome data to be collected, ideally at the end of the follow-up period.

\section{Intensive BP lowering}

Two targets are required for intensive BP lowering:

- Systolic BP $<125 \mathrm{mmHg}$

- Difference in systolic BP between intensive and guideline groups $>10 \mathrm{mmHg}$

Additional guidance on salt and alcohol restriction, and weight reduction is given. The intensive BP treatment algorithm is based on NICE guidelines relating to stroke (CG68 2008 [36]), hypertension (CG127 2011) and type 2 diabetes (CG66 2006, partially updated by CG87 [37]). The algorithm is only a guide and investigators may choose other medications depending on local policy and practice as long as they fit with the overall design of the trial, that is, to achieve intensive BP lowering.

Suitable drug classes and example drugs are:

- ' $\mathrm{A}$ ' = angiotensin converting enzyme inhibitor (ACE-I, for example, perindopril 2 to $8 \mathrm{mg}$ daily (od), ramipril 1.25 to $5 \mathrm{mg}$ twice daily (bd)) or angiotensin receptor antagonist (ARA, for example, losartan 25 to $100 \mathrm{mg}$ od, candesartan 8 to $32 \mathrm{mg}$ od)

- ' $\mathrm{B}$ ' = beta $(\beta)$-receptor antagonist (for example, atenolol 25 to $100 \mathrm{mg}$ od, bisoprolol 5 to $20 \mathrm{mg}$ od)

- ' $C$ ' = calcium channel blocker (for example, amlodipine 5 to $10 \mathrm{mg}$ od, nifedipine LA $30-60 \mathrm{mg}$ od, verapamil SR 120 to $240 \mathrm{mg}$ od) 
- ' $\mathrm{D}$ ' = diuretic (for example, bendroflumethiazide $2.5 \mathrm{mg}$ od, hydrochlorothiazide $12.5 \mathrm{mg}$ od, indapamide $2.5 \mathrm{mg}$ od)

- ' $\mathrm{K}$ ' = potassium-sparing diuretics (for example, spironolactone 12.5 to $100 \mathrm{mg}$ od, amiloride 5 to $20 \mathrm{mg}$ od)

- 'Z' = alpha $(\alpha)$-receptor antagonists (for example, doxazosin 4 to $16 \mathrm{mg}$ od)

- ' $\mathrm{M}$ ' = centrally acting drugs (for example, moxonidine 200 to $600 \mu \mathrm{g}$ daily in divided doses)

In the absence of contraindications, participants should be started on either:

- An 'A' drug, with subsequent addition of a 'C' then 'D' drug (as required)

- A 'C' drug, with subsequent addition of an 'A' then 'D' drug (as required)

If additional treatment is needed to reach target, fourthline and additional options include:

- Add a $\mathrm{K}$ or $\mathrm{Z}$ drug, then the other

- Add an 'M' drug

\section{Intensive lipid lowering}

Two targets are required for intensive lipid lowering:

- Calculated LDL-c $<1.4 \mathrm{mmol} / \mathrm{l}$

- Difference in LDL-c between intensive and guideline groups $>1.0 \mathrm{mmol} / \mathrm{l}$

If LDL-c cannot be calculated (for example, due to an elevated triglyceride level), targets for TC are used instead:

- $\mathrm{TC}<3.1 \mathrm{mmol} / \mathrm{l}$

- Difference in TC between intensive and guideline groups $>1.0 \mathrm{mmol} / \mathrm{l}$

Drug therapy for the intensive lipid arm will typically comprise:

- A third-generation statin (for example, atorvastatin $80 \mathrm{mg}$ od) [38]

- Then add ezetimibe (10 $\mathrm{mg}$ od)

- Then add a resin

Additional guidance on the use of plant stanols/sterols as part of meals, and weight reduction is given. The algorithm for intensive lipid reduction builds on NICE guidelines (CG67, 2008 [39], ezetimibe [40]). Again, the algorithm is only a guide and investigators may choose other treatment strategies depending on local policy and practice as long as they fit with the overall design of the trial, that is, to achieve intensive lipid lowering.

\section{Standard care}

Participants receive standard evidence-based care on top of the interventions, including (as appropriate):

- IS: anticoagulation (cardioembolic stroke), antiplatelets (other IS), carotid endarterectomy

\section{Blood pressure and lipid measurements Blood pressure (BP)}

BP measurements are performed using a validated automated BP monitor, for example, Omron $705 \mathrm{CP}$ or $705 \mathrm{CP}$ II. These devices have been validated by the British Hypertension Society [41], and were used in the positive ASCOT hypertension mega-trial involving 20,000 patients [42]. Baseline and follow-up systolic and diastolic BP and heart rate (HR) readings are taken by trained staff in the nonparetic arm with the participant sitting (three readings) and then standing (one reading).

\section{Ambulatory BP monitoring (ABPM)}

In centers with ABPM equipment (for example, SpaceLabs 90207), participants have 24 hour ABPM performed at baseline and than on treatment every six months. Twentyfour hour, day-time ( 07.00 to 23.00 thrice hourly) and night-time (23.00 to 07.00 hourly) ABPM data are recorded. From these, a number of measures are calculated:

- Mean SBP, DBP and HR for each time interval

- Peak SBP and HR profile over 24 hours

- $\mathrm{BP}$ and HR variation as standard deviation and coefficient of variation $(=\mathrm{SD} /$ Mean $)$

\section{Lipid measurement}

Fasting lipids are measured at an accredited clinical biochemistry laboratory. Fasting should be performed overnight and measurements made at least one month after the last change in lipid lowering therapy. Lipid measurements utilize standard techniques and comprise:

- $\mathrm{TC}$

- Triglyceride (TG)

- HDL cholesterol (HDL-c)

- LDL-c (calculated)

\section{Outcome measure}

Screening

An abbreviated form of the t-MMSE is used to screen patients so that those with dementia are excluded. 


\section{Primary outcome}

The primary outcome is the Addenbrooke's Cognitive Examination-Revised (ACE-R), which includes the MMSE. The ACE- $\mathrm{R}$ is measured at baseline and at each six-month research clinic visit.

\section{Secondary outcomes}

These are assessed at baseline and at each six-month research clinic visit.

- Cognitive outcomes, participant: MoCA, TICS, Stroop and trail-making A and B

- Cognitive assessment, informant: IQCODE

- Cognitive impairment (ACE-R < 89)

- Cognitive decline (reduction in ACE-R by $\geq 10$, or ACE-R < 89)

- Dementia (DSM IV)

- Quality of life: Euro-Qol (EQ-5D and EQ-VAS). A health utility status will be calculated from the EQ-5D using the UK version of the time trade-off algorithm

- Mood: Zung Depression rating Scale (ZDS, short form)

- Function: modified Rankin Scale (mRS), Barthel Index (BI)

- Health resource utilisation: face-to-face survey with participant and carer

- Vascular event: stroke recurrence (by type), myocardial infarction (MI), peripheral arterial disease (PAD)

- Serious adverse events (SAE)

- Disposition: home, home with carer, residential home, nursing home, hospital, death

- Haemodynamics: blood pressure, heart rate

- Blood: fasting lipids (TC, TG, HDL-c, LDL-c)

- Blood: Urea and electrolytes, glucose, HbA1c

A head CT/MR scan is performed once participants have been in the trial at least twelve months (six months minimum). Comparison of this with the index stroke CT scan will allow changes to be identified: new stroke lesions, white matter disease, atrophy.

Dementia, vascular events, and brains scans are adjudicated by experts blinded to treatment assignment. All patients are registered with the Office for National Statistics to identify death and its certified cause.

\section{Sample size calculation Start-up phase}

Recruitment of 600 participants (300 per BP group, approximately, 270 per statin group) will be sufficient to demonstrate adequacy in recruitment of sites and participants, whether sufficient on-treatment differences in BP and lipids can be obtained and maintained, and whether cognition can be assessed satisfactorily. No formal sample size calculation is relevant to this part of the trial.

\section{Main phase}

Using the ACE-R, expanded to include death, as the primary outcome, the whole trial (start-up plus main phases) will need a sample size of 3,400 (1,700 per BP group) poststroke participants, assuming:

- Significance, $\alpha=5 \%$

- Power $(1-\beta)=90 \%$

- Rate of cognitive impairment or death in guideline $\mathrm{BP}$ group $=25 \%$ at five years (main trial, average length of follow-up four years) [34]

- Rate of cognitive impairment or death in 'intensive' $\mathrm{BP}$ group $=20 \%$, that is, absolute risk reduction $(A R R)=5 \%$ (number-needed-to-treat $=20)$, relative risk reduction $(\mathrm{RRR})=20 \%$

- Losses to follow-up $=3 \%$

Hence, 765 participants $(0.225 \times 3,400)$ are anticipated to develop cognitive impairment or die. The sample size allows a smaller but clinically worthwhile decline in cognitive decline to be identified with $80 \%$ power, that is, $\mathrm{ARR}=4.5 \%$ (RRR 18\%). Since there are less existing data on the effect of cholesterol lowering on cognition, the statin factor will assume the same RRR (20\%) but have less power (approximately 86\%) since it will only involve participants with ischemic stroke (approximately 3,060).

Changing from a binary to ordinal analysis of cognitive outcomes may allow for a reduction in sample size of up to $30 \%$, as seen in the 'Optimizing Analysis of Stroke Trials' (OAST) collaboration for functional outcome after stroke [43]. Providing ordinal analysis appears to be more efficient than binary analysis for cognition data, the trial will be re-sized according to the method of Whitehead [44]. Analyses will be adjusted for the covariates since this approach increases statistical power [45] and is recommended by the European Agency for the Evaluation of Medicinal Products (EMEA) Ref CPMP/ EWP/560/98). Any such decision to change will be performed prior to database lock, blinded to treatment, and defined explicitly in the Statistical Analysis Plan.

\section{Statistical analysis}

Feasibility of start-up phase

The feasibility criteria listed in section 2.4.1 are reviewed during the trial. A review at 24 months found that there was no chance of recruiting 600 patients during the internal pilot and that, therefore, the planned main phase should be cancelled (see section 4). 


\section{Comparisons between treatment groups}

Outcomes will be compared between the treatment groups by intention-to-treat (ITT):

- Intensive versus guideline BP lowering

- Intensive versus guideline lipid lowering

Analyses will be adjusted for baseline values and stroke type, age, sex, SBP, TC, and time from stroke to randomization. Continuous covariates (age, SBP, TC, time) will be used with their raw data, that is, not dichotomized. (The full set of stratification and minimization variables listed in section Randomisation will not be used for adjustment because of the limited anticipated sample size of approximately 100).

\section{Missing data, and death}

Missing data will not be imputed. Participants who die will be assigned discrete values for outcome measures with a value worse than any living value (as is standard for mRS, $\mathrm{BI})$. This avoids giving death the same value as the worst possible outcome when alive (best to worst) or, worse, excluding patients who die (since many dementia trials have been confounded by losses to death). Hence, patients who die will be included in all analyses. The EQ-5D Health Utility State (HUS) gives death a score of 0.

- Addenbrooke's Cognitive Examination-Revised (ACE-R), 100 to 0 with death $=-1$

- Mini-Mental State Examination (MMSE), 30 to 0 with death $=-1$

- Telephone-Mini Mental Status Examination ( $\mathrm{t}$-MMSE), 18 to 0 with death $=-1$

- Telephone Interview for Cognitive Status (TICS), 37 to 0 with death $=-1$

- Stroop (accuracy), 24 to 0 with death $=-1$

- Trail-making (accuracy) [46], 25 to 0 with death $=-1$

- Modified Rankin Scale (mRS), 0 to 5 with death $=6$

- Barthel Index (BI), 100 to 0 with death $=-5$

- EuroQol EQ-5D Health Utility State (HUS), 1 to -0.594 with death $=0$

- EuroQol Visual Analog Scale (EQ-VAS), 100 to 0 with death $=-1$.

- Zung Depression Scale (ZDS), 25 to 100 with death $=102.5$

- Verbal fluency (animal naming), $\mathrm{x}$ to 0 , with death $=-1$

\section{Primary outcome}

Comparison of ACE-R (extended to include death - section Missing data, and death) between 'intensive' and 'guideline' BP/lipid lowering groups using multiple linear regression and with adjustment (section Comparisons between treatment groups).

\section{Secondary analyses}

Dichotomous, ordered categorical, continuous and time to event data will be analysed using binary logistic regression (BLR), ordinal logistic regression (OLR), multiple linear regression (MLR) or Cox regression (CR) respectively, and with adjustment (section Comparisons between treatment groups). 95\% confidence intervals will be given and $P<0.05$ will be considered statistically significant.

The proportion of participants with cognitive impairment or who have died, and cognitive decline or died, will be compared between the treatment groups, as done previously for MMSE (a subset of ACE-R) [15,28]. Nevertheless, where possible, continuous or ordinal outcomes will be used in preference to dichotomous outcomes.

\section{Governance and funding}

Trial Steering Committee (TSC)

The TSC provides leadership for the trial and determines and monitors the overall strategy. It meets annually, and has teleconference or Email discussions as needed.

\section{Data Monitoring Committee (DMC)}

The DMC reviews unblinded data annually in respect of safety and efficacy, and considers the study in the context of other trials of dementia prevention post-stroke. It meets at least annually.

\section{Trial Management Committee (TMC)}

The Trial Management Committee (TMC) runs the trial, with meetings every three weeks. It is unblinded to BP and lipid levels, and communicates with the Trial Steering Committee (TSC) and investigators as to whether targets are being met.

\section{Adjudication committees}

All adjudication is performed blinded to treatment assignment:

- Dementia is adjudicated by a group of three individuals (AB, CB, GF); each adjudicator sees each event

- Vascular events are adjudicated by a group of three individuals (PP, AM, RH); each adjudicator sees each event

- Serious adverse events are adjudicated by one of two adjudicators (NS, TE)

\section{Sponsor}

The University of Nottingham is the trial's sponsor.

\section{Funding}

The start-up phase of PODCAST is funded jointly and equally by the UK Alzheimer's Society and UK Stroke Association. 


\section{Trial Status}

A lower than planned recruitment of sites and patients has meant that the aspiration to recruit 600 patients over two years has not been realized. A number of reasons explain the poor recruitment:

- Research governance issues. The trial requires both acute hospital trusts (who identify patients, manage intensive BP and/or lipid lowering, and perform clinic follow-ups) and general practices, through Primary Care Trusts (PCTs, who manage guideline $\mathrm{BP}$ and/or lipid management), to sign-up. In general, each acute trust is associated with between one and three PCTs so that approval for each recruiting site requires between two and four agreements and contracts. It has proved difficult to coordinate the agreement to deliver the study in a locality between these acute and community trusts.

- NHS Excess Treatment Costs. PCTs were often unwilling to approve payment of treatment costs, often citing the cost of atorvastatin (which is now generic but was not so at the start of the trial).

- Long-term follow-up. Once a cohort of patients is recruited, each patient needs follow-up (which

Table 1 Protocol amendments and other changes to trial practice

\begin{tabular}{|c|c|c|c|}
\hline Criterion & Previous versions & Current version/status & Reason \\
\hline \multicolumn{4}{|l|}{ Protocol changes } \\
\hline $\begin{array}{l}\text { Posterior circulation } \\
\text { stroke (POCS) }\end{array}$ & Excluded & Included & $\begin{array}{l}\text { To expand the inclusion criteria; posterior } \\
\text { circulation stroke can lead to cognitive decline }\end{array}$ \\
\hline Exclusion of NYHA 3 or 4 & Exclusion criterion & Removed & To simplify protocol \\
\hline LDL-c target & $<2.0 \mathrm{mmol} / \mathrm{l}$ & $<1.4 \mathrm{mmol} / \mathrm{l}$ & $\begin{array}{l}\text { Half of patients already at } L D L-c<2 \text { at } \\
\text { baseline }\end{array}$ \\
\hline Total cholesterol & $<4.0 \mathrm{mmol}$ & $<3.1 \mathrm{mmol} / \mathrm{l}$ & Ditto \\
\hline Glucose monitoring & & Glucose, $\mathrm{HbA} 1 \mathrm{C}$ & $\begin{array}{l}\text { Some BP and lipid drugs may reduce, or } \\
\text { cause, diabetes mellitus }\end{array}$ \\
\hline Quality of life & DEMQOL & Removed & To simplify protocol \\
\hline Screening & As telephone call & As research clinic visit & To reduce recruitment of ineligible patients \\
\hline $\begin{array}{l}\text { Time from screening } \\
\text { to randomisation }\end{array}$ & 2 weeks & 1 week & To accelerate recruitment \\
\hline Guideline statin dosage & $\begin{array}{l}\text { Simvastatin } 40 \mathrm{mg} \text {, pravastatin } \\
40 \mathrm{mg} \text {, fluvastatin } 40 \mathrm{mg}\end{array}$ & $\begin{array}{l}\text { Simvastatin } 10 \text { to } 40 \mathrm{mg} \text {, pravastatin } \\
10 \text { to } 40 \mathrm{mg} \text {, fluvastatin } 10 \text { to } 80 \mathrm{mg}\end{array}$ & $\begin{array}{l}\text { To reflect NICE guidelines on lipid } \\
\text { management }(C G 67,2008)\end{array}$ \\
\hline \multirow[t]{3}{*}{ Statin classification } & Guideline statin: & Guideline statin: & \multirow{3}{*}{$\begin{array}{l}\text { To clarify intensive versus guideline lipid } \\
\text { lowering management }\end{array}$} \\
\hline & $\begin{array}{l}\text { simvastatin }<40 \mathrm{mg} \text {, pravastatin } \\
\text { any dose, fluvastatin any dose, } \\
\text { atorvastatin } 10 \mathrm{mg} \text {. }\end{array}$ & $\begin{array}{l}\text { simvastatin } \leq 40 \mathrm{mg} \text {, pravastatin any } \\
\text { dose, fluvastatin any dose, } \\
\text { atorvastatin } \leq 20 \mathrm{mg} \text {. }\end{array}$ & \\
\hline & $\begin{array}{l}\text { Intensive statin: } \\
\text { atorvastatin } \leq 40 \mathrm{mg}\end{array}$ & $\begin{array}{l}\text { Intensive statin: atorvastatin > } 20 \text { mg, } \\
\text { rosuvastatin any dose }\end{array}$ & \\
\hline $\begin{array}{l}\text { Trial duration and } \\
\text { participant involvement }\end{array}$ & 8 years & 4 years & $\begin{array}{l}\text { To shorten trial since the main phase is no } \\
\text { longer justified }\end{array}$ \\
\hline $\begin{array}{l}\text { BP and lipid management } \\
\text { in follow-up visits }\end{array}$ & & $\begin{array}{l}\text { 'Floating' visit at any time outside the } \\
\text { planned visits at } 3,6,12,18,24,30 \text {, } \\
36 \text { and } 42 \text { months }\end{array}$ & $\begin{array}{l}\text { To allow enhanced escalation of treatment, as } \\
\text { appropriate }\end{array}$ \\
\hline $\begin{array}{l}\text { Baseline and follow-up BP } \\
\text { and HR monitoring }\end{array}$ & $\begin{array}{l}\text { Three measurements in rapid } \\
\text { succession }\end{array}$ & $\begin{array}{l}\text { Four measurements in rapid } \\
\text { succession including one standing }\end{array}$ & To detect postural hypotension \\
\hline $\begin{array}{l}\text { Neuroimaging sub-study } \\
\text { scan }\end{array}$ & & $\begin{array}{l}\text { CT scan on treatment (plus collection } \\
\text { of any clinical scans during treatment) }\end{array}$ & $\begin{array}{l}\text { To detect potential affects on atrophy, white } \\
\text { matter changes }\end{array}$ \\
\hline Follow-up visits & $\begin{array}{l}\text { Seen in clinic once a year with } \\
\text { interval blinded telephone } \\
\text { follow-up. }\end{array}$ & Seen in clinic once every 6 months & $\begin{array}{l}\text { To assess latest BP and/or lipid levels and } \\
\text { escalate treatment as appropriate }\end{array}$ \\
\hline \multicolumn{4}{|l|}{ Other changes } \\
\hline Minimisation variables & $\begin{array}{l}\text { As in section Minimisation (on } \\
\text { key prognostic/logistical } \\
\text { variables) above }\end{array}$ & Age, systolic BP, LDL-C & $\begin{array}{l}\text { Small trial size precluded numerous } \\
\text { minimisation variables }\end{array}$ \\
\hline Email reminders & & Twice yearly to investigators. & $\begin{array}{l}\text { To highlight the need to achieve targets in BP } \\
\text { and lipid lowering in patients randomised to } \\
\text { intensive management }\end{array}$ \\
\hline
\end{tabular}


typically last two and a half hours) every six months, thereby placing a considerable work load on research staff at acute sites.

- Changes to the original protocol are summarised in Table 1

\section{Abbrevations}

PODCAST: Prevention of Decline in Cognition after Stroke Trial;

TICS: Telephone Interview for Cognitive Status; MOCA: Montreal Cognitive Assessment; ACE-R: Addenbrooke's Cognitive Examintaion-revised; MMSE: Mini-Mental State Examination; od: once daily; bd: Twice daily; PCT: Primary Care Trust; IQCODE: Informant Questionnaire on Cognitive Decline in the Elderly; DSM IV: Diagnostic and Statistical Manual of Mental Disorders edition IV; BP: Blood pressure; SBP: Systolic Blood Pressure; DBP: Diastolic Blood Pressure; ABPM: Ambulatory Blood Pressure Monitoring; HR: Heart rate; MI: Myocardial infarction; PAD: Peripheral arterial disease; eGFR: estimated Glomerular Filtration Rate; ALT: ALanine Transaminase; IS: Ischaemic stroke; ICH: Intracerebral haemorrhage; SAE: Serious adverse events; GP: General practitioner; WMH: White matter hyperintensities; POCS: Posterior Circulation Stroke; ARR: Absolute risk reduction; NNT: Number-needed-to-treat; RRR: Relative risk reduction; ITT: Intention-totreat; CT: Computed Tomography; MR: Magnetic Resonance imaging; TC: Total cholesterol; TG: Triglycerides; HDL-c: High Density Lipoprotein cholesterol; LDL-c: Low Density Lipoprotein cholesterol; OAST: Optimizing Analysis of Stroke Trials'; EMEA: European Agency for the Evaluation of Medicinal Products; BLR: Binary logistic regression; OLR: Ordinal logistic regression; MLR: Multiple linear regression; CR: Cox regression; mRS: Modified Rankin Scale; BI: Barthel Index; HUS: EuroQol EQ-5D Health Utility State; ZDS: Zung Depression Scale; TMC: Trial Management Committee; TSC: Trial Steering Committee; DMC: Data Monitoring Committee.

\section{Competing interests}

The authors declare that they have no competing interests.

\section{Authors' contributions}

DJB and PB drafted the manuscript and updated the current literature for the background section. Statistical advice was provided by SP. CB, PB, AB, GF, $J M, P P, S P, J R, R S$ and JW designed the study and wrote the protocol. All authors read and improved the final manuscript.

\section{Acknowledgements}

\section{Trial steering committee}

Independent experts: John O'Brien (TSC Chair, Cambridge), Stephen Ball (Cardiology, Leeds).

Grant holders: Philip Bath (Stroke, Chief Investigator - lipid lowering arm, Nottingham), Gary Ford (Stroke, Chief Investigator - BP lowering arm, Newcastle), Clive Ballard (Psychiatry, London), Alistair Burns (Psychiatry, Manchester), Jonathan Mant (Public Health, Cambridge), Peter Passmore (Geriatrics, Belfast), John Reckless (Lipids, Bath), Rob Stewart (Psychiatry, London), Joanna Wardlaw (Neuroradiology, Edinburgh), Nikola Sprigg (Stroke, Nottingham).

Patient and Carer representatives: Dave Hanbury, John Murray, Andrew Pepper

Funders' representative: Susanne Sorensen/James Pickett (Alzheimer's Society), Peter Coleman (The Stroke Association).

Sponsor representative: Angela Shone (University of Nottingham).

\section{Data monitoring committee}

John Geddes (Chair, Oxford), Jan Staessen (Hypertension, Leuven), Chris Weir (Statistics, Edinburgh), Lydia Fox (non-voting, statistics, Nottingham).

\section{Cognition/dementia adjudicators}

Alistair Burns (Chair, Psychiatry, Manchester), Clive Ballard (Psychiatry, London), Gary Ford (Stroke, Newcastle).

\section{Vascular events adjudicators}

Peter Passmore (Chair, Geriatrics, Belfast), Amit Mistri (Stroke, Leicester), Rob Henderson (Cardiology, Nottingham).
Serious adverse event adjudicator

Nikola Sprigg (Stroke, Nottingham), Tim England (Stroke, Derby/Nottingham).

\section{Neuroimaging}

Joanna Wardlaw (Chair, Neuroradiology, Edinburgh), Dan Blackburn

(Neurology, Sheffield).

\section{Health economics}

Paul McCrone, Martin Knapp (London).

\section{Statisticians}

Stuart Pocock (Consultant, London), Lydia Fox (Analyst, Nottingham).

\section{Programming/database management}

Lee Haywood, Liz Walker, Richard Dooley (Nottingham).

\section{Sponsor}

University of Nottingham.

\section{Participating sites (who have given research ethics approval)}

Aberdeen Royal Infirmary (NHS Grampian).

Aintree Hospitals NHS Foundation Trust.

Birmingham Heartlands \& Solihull Hospitals NHS Trust.

Bradford Teaching Hospitals NHS Foundation Trust.

Chesterfield Royal Hospital NHS Foundation Trust.

Derby Hospitals NHS Foundation Trust.

Doncaster \& Bassetlaw Hospitals NHS Foundation Trust.

Mid Cheshire Hospitals NHS Foundation Trust.

North Cumbria University Hospitals NHS Trust.

Newcastle-Upon-Tyne Hospitals NHS Foundation Trust.

North Tees and Hartlepool NHS Foundation Trust.

Nottingham University Hospitals NHS Trust.

Poole Hospital NHS Foundation Trust.

Queen Elizabeth Queen Mother Hospital, Margate.

Royal Cornwall Hospitals NHS Trust.

Royal United Hospital Bath NHS Trust.

Royal Bournemouth \& Christchurch Hospitals NHS Trust.

South Tees Hospitals NHS Foundation Trust.

South Tyneside NHS Foundation Trust.

University Hospitals of Leicester NHS Trust.

Yeovil District Hospital NHS Foundation Trust.

York Hospitals NHS Foundation Trust.

\section{Funding}

Jointly and equally by the UK Alzheimer's Society and UK Stroke Association.

\section{Author details}

'Sheffield Institute for Translational Neuroscience, University of Sheffield, 385A Glossop Road, Sheffield S10 2HQ, UK. ${ }^{2}$ Stroke Trials Unit, Division of Clinical Neuroscience, University of Nottingham, City Hospital campus, Nottingham NG5 1PB, UK. ${ }^{3}$ Wolfson Centre for Age-Related Diseases, Wolfson Wing, Hodgkin Building, King's College London, Guy's Campus, London SE1 IUL, UK. ${ }^{4}$ Faculty of Medical and Human Sciences, Institute of Brain, Behavior and Mental Health, University of Manchester, Grafton Street, Manchester M13 9NT, UK. ${ }^{5}$ Level 6, Leazes Wing, Royal Victoria Infirmary, Newcastle NE14 LP5, UK. ${ }^{6}$ General Practice \& Primary Care Research Unit, University of Cambridge, Addenbrooke's Hospital, Forvie Site, Cambridge CB2 OSR, UK. ${ }^{7}$ Institute of Clinical Sciences, Queens University, Belfast, Royal Victoria Hospital, Block B, Belfast BT12 6BA, UK. ${ }^{8}$ Department of Medical Statistics, London School of Hygiene and Tropical Medicine, Keppel Street, London WC1E 7HT, UK. ${ }^{9}$ Department of Endocrinology, Royal United Hospital, Combe Park, Bath BA1 3NG, UK. ${ }^{10}$ Section of Epidemiology (Box 60), Institute of Psychiatry (King's College London), De Crespigny Park, London SE5 8AF, UK. "'Division of Clinical Neurosciences, Western General Hospital, Crewe Rd, Edinburgh EH4 2XU, UK.

Received: 24 July 2013 Accepted: 28 October 2013

Published: 22 November 2013 


\section{References}

1. Pendlebury ST, Rothwell PM: Prevalence, incidence, and factors associated with pre-stroke and post-stroke dementia: a systematic review and meta-analysis. Lancet Neurol 2009, 8(11):1006-1018.

2. Gottesman RF, Hillis AE: Predictors and assessment of cognitive dysfunction resulting from ischemic stroke. Lancet Neurol 2010, 9(9):895-905.

3. Wiberg B, et al: The relationship between executive dysfunction and post-stroke mortality: a population-based cohort study. BMJ Open 2012, 2(3):e000458.

4. Melkas S, et al: Depression-executive dysfunction syndrome relates to poor poststroke survival. Am J Geriatr Psychiatry 2010, 18(11):1007-1016

5. Nys GM, et al: The prognostic value of domain-specific cognitive abilities in acute first-ever stroke. Neurology 2005, 64(5):821-827.

6. Tham W, et al: Progression of cognitive impairment after stroke: one year results from a longitudinal study of Singaporean stroke patients. J Neurol Sci 2002, 203-204:49-52.

7. Pasquier F, Henon H, Leys D: Relevance of white matter changes to pre- and poststroke dementia. Ann N Y Acad Sci 2000, 903:466-469.

8. Stebbins GT, et al: Gray matter atrophy in patients with ischemic stroke with cognitive impairment. Stroke 2008, 39(3):785-793.

9. Collaborating PATS: Group: Post-stroke antihypertensive treatment study. A preliminary result. Chin Med J 1995, 108:710-717.

10. PROGRESS Collaborative Group: Randomized trial of a perindoprilbased blood-pressure-lowering regimen among 6,105 individuals with previous stroke or transient ischemic attack. Lancet 2001, 358:1033-1041.

11. Rashid PA, Leonardi-Bee J, Bath PM: Lowering blood pressure after stroke or transient ischemic attack to prevent recurrence: a systematic review of randomized controlled trials. Cerebrovasc Dis 2002, 13(suppl 3):92. abstract.

12. Skoog l, et al: 15-year longitudinal study of blood pressure and dementia. Lancet 1996, 347(9009):1141-1145.

13. Petrovitch $\mathrm{H}$, et al: Midlife blood pressure and neuritic plaques, neurofibrillary tangles, and brain weight at death: the HAAS. Honolulu-Asia aging Study. Neurobiol Aging 2000, 21(1):57-62.

14. Launer $\sqcup$, et al: Midlife blood pressure and dementia: the Honolulu-Asia aging study. Neurobiol Aging 2000, 21(1):49-55.

15. Tzourio C, et al: Effects of blood pressure lowering with perindopril and indapamide therapy on dementia and cognitive decline in patients with cerebrovascular disease. Arch Intern Med 2003, 163(9):1069-1075

16. Diener $\mathrm{H}-\mathrm{C}$, et al: Effects of aspirin plus extended-release dipyridamole versus clopidogrel and telmisartan on disability and cognitive function after recurrent stroke in patients with ischemic stroke in the Prevention Regimen for Effectively Avoiding Second Strokes (PRoFESS) trial: a double-blind, active and placebo-controlled study. Lancet Neurol 2008, 7(10):875-884

17. Peters $\mathrm{R}$, et al: Incident dementia and blood pressure lowering in the Hypertension in the Very Elderly Trial cognitive function assessment (HYVET-COG): a double-blind, placebo controlled trial. Lancet Neurol 2008, 7(8):683-9.

18. Forette $F$, et al: Prevention of dementia in randomized double-blind placebo-controlled Systolic Hypertension in Europe (Syst-Eur) trial. Lancet 1998, 352:1347-1351.

19. Ankolekar $\mathrm{S}$, et al: Clinical trials for preventing post stroke cognitive impairment. J Neurol Sci 2010, 299(1-2):168-174.

20. SPS3 Investigators BO, Hart RG, McClure LA, Szychowski JM, Coffey CS, Pearce LA: Effects of clopidogrel added to aspirin in patients with recent lacunar stroke. Engl J Med 2012, 376(9):817-825.

21. Jacova C, et al: Cognitive impairment in lacunar strokes: the SPS3 trial. Ann Neurol 2012, 72(3):351-362.

22. Steiner $S$, et al: Simvastatin blunts endotoxin-induced tissue factor in vivo. Circulation 2005, 111(14):1841-1846.

23. Ii M, Losordo DW: Statins and the endothelium. Vascul Pharmacol 2007, 46(1):1-9.

24. Snowdon DA, et al: Brain infarction and the clinical expression of Alzheimer disease. The Nun Study. JAMA 1997, 277(10):813-817.

25. Heart Protection Study Collaborative Group: Effects of cholesterol-lowering with simvastatin on stroke and other major vascular events in 20,536 people with cerebrovascular disease or other high-risk conditions. Lancet 2004, 363(9411):757-767.

26. The Stroke Prevention by Aggressive Reduction in Cholesterol Levels (SPARCL) Investigators: High-dose atorvastatin after stroke or transient ischemic attack. Engl J Med 2006, 355(6):549-559.

27. Manktelow BN, Potter JF: Interventions in the management of serum lipids for preventing stroke recurrence. Cochrane Database Syst Rev 2009, 8(3), CD002091.

28. HPS: MRC/BHF Heart Protection Study of cholesterol lowering with simvastatin in 20,536 high-risk individuals: a randomized placebo-controlled trial. Lancet 2002, 360(9326):7-22.

29. Shepherd J, et al: Pravastatin in elderly individuals at risk of vascular disease (PROSPER): a randomized controlled trial. Lancet 2002, 360(9346):1623-1630.

30. Golomb BA, Evans MA: Statin adverse effects: a review of the literature and evidence for a mitochondrial mechanism. Am J Cardiovasc Drugs 2008, 8(6):373-418.

31. Bamford J, et al: Classification and natural history of clinically identifiable subtypes of cerebral infarction. Lancet 1991, 337:1521-1526.

32. Adams HP, et al: Classification of subtype of acute ischemic stroke. Definitions for use in a multicenter clinical trial. Stroke 1993, 24:35-41.

33. Lenzi GL, Altieri M: Short-term evolution as a marker of vascular dementia versus Alzheimer's disease. J Neurol Sci 2007, 257(1-2):182-184

34. Jorm AF, Jacomb PA: The informant questionnaire on cognitive decline in the elderly (IQCODE): socio-demographic correlates, reliability, validity and some norms. Psycholog Med 1989, 19:1015-1022.

35. Weir CJ, Lees KR: Comparison of stratification and adaptive methods for treatment allocation in an acute stroke clinical trial. Stat Med 2003, 22:705-726.

36. Group, TGD: Stroke: diagnosis and initial management of acute stroke and transient ischemic attack. NICE Clin Guidel 2008, CG68

37. Group TGD: Type 2 diabetes. The management of type 2 diabetes. NICE Clin Guidel 2009, 87.

38. Amarenco $\mathrm{P}$, et al: High-dose atorvastatin after stroke or transient ischemic attack. N Engl J Med 2006, 355(6):549-559.

39. Cooper A: O'Flynn N, on behalf of the Guideline Development Group: Risk assessment and lipid modification for primary and secondary prevention of cardiovascular disease: summary of NICE guidance. BMJ 2008, 336:1246-1248.

40. Group TGD: Ezetimibe for the treatment of primary (heterozygousfamilial and non-familial) hypercholesterolemia. NICE Technol Appr Guid 2007, 132.

41. O'Brien E, Atkins N: A comparison of the British Hypertension Society and Association for the Advancement of Medical Instrumentation protocols for validating blood pressure measuring devices: can the two be reconciled? J Hypertens 1994, 12(9):1089-1094.

42. Dahlof $B$, et al: Prevention of cardiovascular events with an antihypertensive regimen of amlodipine adding perindopril as required versus atenolol adding bendroflumethiazide as required, in the AngloScandinavian Cardiac Outcomes Trial-Blood Pressure Lowering Arm (ASCOT-BPLA): a multicenter randomized controlled trial. Lancet 2005, 366(9489):895-906

43. The Optimizing Analysis of Stroke Trials (OAST) Collaboration: Can we improve the statistical analysis of stroke trials? Statistical re-analysis of functional outcomes in stroke trials. Stroke 2007, 38:1911-1915.

44. The Optimizing Analysis of Stroke Trials (OAST) Collaboration: Calculation of sample size for stroke trials assessing functional outcome: comparison of binary and ordinal approaches. Int I Stroke 2008, 3:78-84.

45. The Optimizing Analysis of Stroke Trials (OAST) Collaboration: Should stroke trials adjust functional outcome for baseline prognostic factors? Stroke 2009, 40:888-894.

46. Bowie CR HP: Administration and interpretation of the Trail Making Test. Nat Proc 2006, 1(5):2277-2281.

doi:10.1186/1745-6215-14-401

Cite this article as: Blackburn et al:: Prevention of Decline in Cognition after Stroke Trial (PODCAST): a study protocol for a factorial randomised controlled trial of intensive versus guideline lowering of blood pressure and lipids. Trials 2013 14:401. 